\title{
A study of second-order $q$-difference equations with boundary conditions
}

Bashir Ahmad ${ }^{1 *}$, Ahmed Alsaedi ${ }^{1}$ and Sotiris $\mathrm{K}_{\text {Ntouyas }}{ }^{2}$

* Correspondence:

bashir_qau@yahoo.com

'Department of Mathematics,

Faculty of science, King Abdulaziz

University, P.O. Box 80203, Jeddah

21589, Saudi Arabia

Full list of author information is

available at the end of the article

\begin{abstract}
This paper studies a boundary value problem of nonlinear second-order q-difference equations with non-separated boundary conditions. As a first step, the given boundary value problem is converted to an equivalent integral operator equation by using the q-difference calculus. Then the existence and uniqueness of solutions of the problem is proved via the resulting integral operator equation by means of Leray-Schauder nonlinear alternative and some standard fixed point theorems. Our approach is simpler than the one involving the typical series solution form of $q^{-}$ difference equations. The results corresponding to a second-order q-difference equation with anti-periodic boundary conditions appear as a special case.

Furthermore, our results reduce to the corresponding results for classical secondorder boundary value problems with non-separated boundary conditions in the limit $q \rightarrow 1$, which provides a useful check.

2010 Mathematics Subject Classification. 39A05, 39A13.
\end{abstract}

Keywords: q-difference equations, non-separated boundary conditionss, existence, fixed point

\section{Introduction}

In this paper, we discuss the existence of solutions for the second-order $q$-difference equation with non-separated boundary conditions

$$
\left\{\begin{array}{c}
D_{q}^{2} u(t)=f(t, u(t)), t \in I, \\
u(0)=\eta u(T), D_{q} u(0)=\eta D_{q} u(T),
\end{array}\right.
$$

where $f \in C(I \times \mathbb{R}, \mathbb{R}), I=[0, T] \cap q^{\overline{\mathbb{N}}}, q^{\overline{\mathbb{N}}}:=\left\{q^{n}: n \in \mathbb{N}\right\} \cup\{0\}$ and $T \in q^{\overline{\mathbb{N}}}$ is a fixed constant, and $\eta \neq 1$ is a fixed real number.

The study of $q$-difference equations, initiated in the beginning of the 20th century ([1-4]), and, up to date, it has evolved into a multidisciplinary subject, (for example, see ([5-15]) and references therein). For some recent work on $q$-difference equations, we refer the reader to the papers ([16-23]). However, the theory of boundary value problems for nonlinear $q$-difference equations is still in the initial stage and many aspects of this theory need to be explored.

The main objective of this paper is to develop some existence and uniqueness results for the boundary value problem (1.1). Our results are based on a variety of fixed point theorems such as Banach's contraction principle, Leray-Schauder nonlinear alternative

(C) 2012 Ahmad et al.; licensee Springer. This is an open access article distributed under the terms of the Creative Commons Attribution License (http://creativecommons.org/licenses/by/2.0), which permits unrestricted use, distribution, and reproduction in any medium, provided the original work is properly cited. 
and Krasnoselskii's fixed point theorem. Some illustrative examples and special cases are also discussed.

\section{Preliminaries}

Let us recall some basic concepts of $q$-calculus $[14,15]$.

For $0<q<1$, we define the $q$-derivative of a real valued function $f$ as

$$
D_{q} f(t)=\frac{f(t)-f(q t)}{(1-q) t}, D_{q} f(0)=\lim _{t \rightarrow 0} D_{q} f(t) .
$$

The higher order $q$-derivatives are given by

$$
D_{q}^{0} f(t)=f(t), \quad D_{q}^{n} f(t)=D_{q} D_{q}^{n-1} f(t), \quad n \in \mathbb{N} .
$$

The q-integral of a function $f$ defined in the interval $J_{a}=[a, b] \cap q^{\overline{\mathbb{N}}}$ is given by

$$
\int_{a}^{x} f(t) d_{q} t:=\sum_{n=0}^{\infty}(1-q) q^{n}\left[x f\left(x q^{n}\right)-a f\left(q^{n} a\right)\right], \quad x \in J_{a}
$$

and for $a=0$, we denote

$$
I_{q} f(x)=\int_{0}^{x} f(t) d_{q} t=\sum_{n=0}^{\infty} x(1-q) q^{n} f\left(x q^{n}\right),
$$

provided the series converges. If $a \in J_{0}=[0, b] \cap q^{\overline{\mathbb{N}}}$ and $f$ is defined in the interval $J_{0}$, then

$$
\int_{a}^{b} f(t) d_{q} t=\int_{0}^{b} f(t) d_{q} t-\int_{0}^{a} f(t) d_{q} t
$$

Similarly, we have

$$
I_{q}^{0} f(t)=f(t), \quad I_{q}^{n} f(t)=I_{q} I_{q}^{n-1} f(t), \quad n \in \mathbb{N} .
$$

Observe that

$$
D_{q} I_{q} f(x)=f(x),
$$

and if $f$ is continuous at $x=0$, then

$$
I_{q} D_{q} f(x)=f(x)-f(0) .
$$

In $q$-calculus, the product rule and integration by parts formula are

$$
\begin{aligned}
& D_{q}(g h)(t)=\left(D_{q} g(t)\right) h(t)+g(q t) D_{q} h(t), \\
& \int_{0}^{x} f(t) D_{q} g(t) d_{q} t=[f(t) g(t)]_{0}^{x}-\int_{0}^{x} D_{q} f(t) g(q t) d_{q} t .
\end{aligned}
$$

In the limit $q \rightarrow 1$ the above results correspond to their counterparts in standard calculus.

Lemma 2.1 The unique solution of (1.1) is given by

$$
u(t)=\int_{0}^{t}(t-q s) f(s, u(s)) d_{q} s+\frac{\eta}{(\eta-1)^{2}} \int_{0}^{T}[T+(1-\eta)(t-q s)] f(s, u(s)) d_{q} s .
$$


Proof. Integrating the equation $D_{q}^{2} u=f(t, u)$, we get

$$
D_{q} u(t)=\int_{0}^{t} f(s, u(s)) d_{q} s+a_{1} .
$$

Integrating (2.5), we obtain

$$
u(t)=\int_{0}^{t}\left(\int_{0}^{v} f(s, u(s)) d_{q} s\right) d_{q} v+a_{1} t+a_{2}
$$

which, on changing the order of integration, takes the form

$$
u(t)=\int_{0}^{t}\left(\int_{q s}^{t} f(s, u(s)) d_{q} v\right) d_{q} s+a_{1} t+a_{2} .
$$

Alternatively, (2.7) can be written as

$$
u(t)=\int_{0}^{t}(t-q s) f(s, u(s)) d_{q} s+a_{1} t+a_{2} .
$$

Here $a_{1}, a_{2}$ are arbitrary constants. Conversely, differentiating (2.8) by applying the formulae (2.1) and (2.2) of $q$-calculus, one can easily obtain $D_{q}^{2} u=f(t, u)$.

Now, using the boundary conditions of (1.1) in (2.8), we find that

$$
\begin{aligned}
& a_{1}=\frac{-\eta}{(\eta-1)} \int_{0}^{T} f(s, u(s)) d_{q} s \\
& a_{2}=\frac{\eta^{2} T}{(\eta-1)^{2}} \int_{0}^{T} f(s, u(s)) d_{q} s-\frac{\eta}{(\eta-1)} \int_{0}^{T}(T-q s) f(s, u(s)) d_{q} s .
\end{aligned}
$$

Substituting the values of $a_{1}$ and $a_{2}$ in (2.8), we obtain (2.4). This completes the proof.

For the forthcoming analysis, let $\mathcal{C}=C(I, \mathbb{R})$ denotes the Banach space of all continuous functions from $I$ to $\mathbb{R}$ endowed with the norm defined by $\|x\|=\sup \{|x(t)|, t \in I\}$.

Furthermore, we set

$$
\begin{aligned}
\Lambda_{1} & =\sup _{t \in I}\left\{\frac{t^{2}}{1+q}+\frac{|\eta(1+\eta q)| T^{2}}{(1+q)(\eta-1)^{2}}+\left|\frac{\eta}{\eta-1}\right| t T\right\} \\
& =\left(\frac{1}{1+q}+\frac{|\eta(1+\eta q)|}{(1+q)(\eta-1)^{2}}+\left|\frac{\eta}{\eta-1}\right|\right) T^{2} .
\end{aligned}
$$

Theorem 2.1 Let $f: I \times \mathbb{R} \rightarrow \mathbb{R}$ be a continuous function satisfying the condition

$$
\left|\left(H_{0}\right)\right| f(t, u)-f(t, v)|\leq L| u-v \mid, \forall t \in I, u, v \in \mathbb{R},
$$

where $L$ is a Lipschitz constant. Then the boundary value problem (1.1) has a unique solution, provided $\Lambda=L \Lambda_{1}<1$, where $\Lambda_{1}$ is given by (2.9).

Proof. We define $\digamma: \mathcal{C} \rightarrow \mathcal{C}$ by

$$
\digamma u(t)=\int_{0}^{t}(t-q s) f(s, u(s)) d_{q} s+\frac{\eta}{(\eta-1)^{2}} \int_{0}^{T}[T+(1-\eta)(t-q s)] f(s, u(s)) d_{q} s
$$


for $u \in \mathcal{C}$ and $t \in I$. Let us set $\sup _{t \in I}|f(t, 0)|=M_{0}$ and choose

$$
r \geq \frac{M_{0} \Lambda_{1}}{1-\delta}
$$

where $\delta$ is such that $\Lambda \leq \delta<1$. Now we show that $\digamma B_{r} \subset B_{r}$, where $B_{r}=\{u \in \mathcal{C}:\|u\| \leq r\}$. For $u \in B_{r}$, we have

$$
\begin{aligned}
\|(\digamma u)(t)\|= & \sup _{t \in I} \mid \int_{0}^{t}(t-q s) f(s, u(s)) d_{q} s \\
& +\frac{\eta}{(\eta-1)^{2}} \int_{0}^{T}[T+(1-\eta)(t-q s)] f(s, u(s)) d_{q} s \mid \\
\leq & \sup _{t \in I} \mid \int_{0}^{t}(t-q s)(|f(s, u(s))-f(s, 0)|+|f(s, 0)|) d_{q} s \\
& +\frac{\eta}{(\eta-1)^{2}} \int_{0}^{T}[T+(1-\eta)(t-q s)](|f(s, u(s))-f(s, 0)|+|f(s, 0)|) d_{q} s \mid \\
\leq & \sup _{t \in I} \mid \int_{0}^{t}(t-q s)(L|u(s)|+|f(s, 0)|) d_{q} s \\
& +\frac{\eta}{(\eta-1)^{2}} \int_{0}^{T}[T+(1-\eta)(t-q s)](L|u(s)|+|f(s, 0)|) d_{q} s \mid \\
\leq & \left(L\|u\|+M_{0}\right) \sup _{t \in I} \mid \int_{0}^{t}(t-q s) d_{q} s \\
& +\frac{\eta}{(\eta-1)^{2}} \int_{0}^{T}[T+(1-\eta)(t-q s)] d_{q} s \mid \\
\leq & \left(L\|u\|+M_{0}\right) \sup _{t \in I}\left\{\frac{t^{2}}{1+q}+\frac{|\eta(1+\eta q)| T^{2}}{(1+q)(\eta-1)^{2}}+\left|\frac{\eta}{\eta-1}\right| t T\right\} \\
\leq & \Lambda_{1}\left(L r+M_{0}\right) \leq(\Lambda+1-\delta) r \leq r .
\end{aligned}
$$

Now, for $u, v \in \mathcal{C}$ and for each $t \in I$, we obtain

$$
\begin{aligned}
& \|(\digamma u)(t)-(\digamma v)(t)\| \\
= & \sup _{t \in I}|(\digamma u)(t)-(\digamma v)(t)| \\
\leq & \sup _{t \in I} \mid \int_{0}^{t}(t-q s)[f(s, u(s))-f(s, v(s))] d_{q} s \\
& +\frac{\eta}{(\eta-1)^{2}} \int_{0}^{T}[T+(1-\eta)(t-q s)][f(s, u(s))-f(s, v(s))] d_{q} s \mid \\
\leq & L \sup _{t \in I}\left|\int_{0}^{t}(t-q s) d_{q} s+\frac{\eta}{(\eta-1)^{2}} \int_{0}^{T}[T+(1-\eta)(t-q s)] d_{q} s\right|\|u-v\| \\
\leq & L \sup _{t \in I}\left\{\frac{t^{2}}{1+q}+\frac{|\eta(1+\eta q)| T^{2}}{(1+q)(\eta-1)^{2}}+\left|\frac{\eta}{\eta-1}\right| t T\right\}\|u-v\| \\
\leq & \Lambda\|u-v\|,
\end{aligned}
$$

where

$$
\Lambda=L\left(\frac{1}{1+q}+\frac{|\eta(1+\eta q)|}{(1+q)(\eta-1)^{2}}+\left|\frac{\eta}{\eta-1}\right|\right) T^{2},
$$


which depends only on the parameters involved in the problem. As $\Lambda<1$, therefore

$\nabla$ is a contraction. Thus, the conclusion of the theorem follows by Banach's contraction mapping principle. This completes the proof.

The next existence result is based on Leray-Schauder alternative.

Theorem 2.2 (Nonlinear alternative for single valued maps)[24]. Let $E$ be a Banach space, $C$ a closed, convex subset of $E, U$ an open subset of $C$ and $\mathrm{O} L U$. Suppose that $F$ : $\bar{U} \rightarrow C$ is a continuous, compact (that is, $F(\bar{U})$ is a relatively compact subset of $C$ ) map. Then either

(i) F has a fixed point in $\bar{U}$, or

(ii) there is a $u \in \partial U$ (the boundary of $U$ in $C$ ) and $\lambda \in(0,1)$ with $u=\lambda F(u)$.

Theorem 2.3 Assume that:

$\left(H_{1}\right)$ there exists a continuous nondecreasing function $\psi:[0, \infty) \rightarrow(0, \infty)$ and a function $p \in L^{1}\left(I, \mathbb{R}_{+}\right)$such that

$$
|f(t, u)| \leq p(t) \psi(|u|) \text { for each }(t, u) \in I \times \mathbb{R} ;
$$

$\left(H_{2}\right)$ there exists a number $M<0$ such that

$$
\frac{M}{T\left(1+\frac{|\eta|(1+|1-\eta|)}{(\eta-1)^{2}}\right) \psi(M)\|p\| L^{1}}>1,
$$

where

$$
\|p\|_{L^{1}}=\int_{0}^{T} p(s) d_{q} s \neq 0 .
$$

Then the BVP (1.1) has at least one solution.

Proof. We define $\digamma: \mathcal{C} \rightarrow \mathcal{C}$ as in (2.10). The proof consists of several steps.

(i) $F$ maps bounded sets into bounded sets in $C(I, \mathbb{R})$.

Let $B_{k}=\{u \in C(I, \mathbb{R}):\|u\| \leq k\}$ be a bounded set in $C(I, \mathbb{R})$ and $u \in B_{k}$. Then we have

$$
\begin{aligned}
|\digamma u(t)| & \leq \int_{0}^{t}|t-q s||f(s, u(s))| d_{q} s+\frac{|\eta|}{(\eta-1)^{2}} \int_{0}^{T}|T+(1-\eta)(t-q s)||f(s, u(s))| d_{q} s \\
& \leq T\left(1+\frac{|\eta|(1+|1-\eta|)}{(\eta-1)^{2}}\right) \int_{0}^{T} p(s) \psi(\|u\|) d_{q} s \\
& \leq T\left(1+\frac{|\eta|(1+|1-\eta|)}{(\eta-1)^{2}}\right) \psi(k) \int_{0}^{T} p(s) d_{q} s .
\end{aligned}
$$

Thus

$$
\|\digamma u\| \leq T\left(1+\frac{|\eta|(1+|1-\eta|)}{(\eta-1)^{2}}\right) \psi(k)\|p\|_{L^{1}} .
$$

(ii) F maps bounded sets into equicontinuous sets of $C(I, \mathbb{R})$.

Let $r_{1}, r_{2} \in I, r_{1}<r_{2}$ and $B_{k}$ be a bounded set of $C(I, \mathbb{R})$ as before. Then for $u \in B_{k}$ we have 


$$
\begin{aligned}
\left|\digamma u\left(r_{2}\right)-\digamma u\left(r_{1}\right)\right|= & \mid \int_{0}^{r_{2}}\left(r_{2}-q s\right) f(s, u(s)) d_{q} s-\int_{0}^{r_{1}}\left(r_{1}-q s\right) f(s, u(s)) d_{q} s \\
& +\frac{\eta(1-\eta)}{(\eta-1)^{2}} \int_{0}^{T}\left(r_{2}-r_{1}\right) f(s, u(s)) d_{q} s \mid \\
= & \mid \int_{0}^{r_{1}}\left(r_{2}-r_{1}\right) f(s, u(s)) d_{q} s+\int_{r_{1}}^{r_{2}}\left(r_{2}-q s\right) f(s, u(s)) d_{q} s \\
& +\frac{\eta(1-\eta)}{(\eta-1)^{2}} \int_{0}^{T}\left(r_{2}-r_{1}\right) f(s, u(s)) d_{q} s \mid \\
\leq & \int_{0}^{r_{1}}\left|r_{2}-r_{1}\right| p(s) \psi(k) d_{q} s+\int_{r_{1}}^{r_{2}}\left|r_{2}-q_{s}\right| p(s) \psi(k) d_{q} s \\
& +\frac{|\eta|}{|1-\eta|} \int_{0}^{T}\left|r_{2}-r_{1}\right| p(s) \psi(k) d_{q} s .
\end{aligned}
$$

The right hand side tends to zero as $r_{2}-r_{1} \rightarrow 0$. As a consequence of Arzelá-Ascoli theorem, we can conclude that $\digamma: C(I, \mathbb{R}) \longrightarrow C(I, \mathbb{R})$ is completely continuous.

(iii) Let $\lambda \in(0,1)$ and let $u=\lambda \digamma u$. Then, for $t \in I$, we have

$$
\begin{aligned}
|u(t)|= & |\lambda \digamma u(t)| \leq \int_{0}^{t}|t-q s||f(s, u(s))| d_{q} s \\
& +\frac{|\eta|}{(\eta-1)^{2}} \int_{0}^{T}|T+(1-\eta)(t-q s)||f(s, u(s))| d_{q} s \\
\leq & T\left(1+\frac{|\eta|(1+|1-\eta|)}{(\eta-1)^{2}}\right) \psi(\|u\|) \int_{0}^{T} p(s) d,
\end{aligned}
$$

and consequently

$$
\frac{\|u\|}{T\left(1+\frac{|\eta|(1+|1-\eta|)}{(\eta-1)^{2}}\right) \psi(\|u\|)\|p\|_{L^{1}}} \leq 1 .
$$

In view of $\left(H_{2}\right)$, there exists $M$ such that $\|u\| \neq M$. Let us set

$$
U=\{u \in C(I, \mathbb{R}):\|u\|<M\} .
$$

Note that the operator $\digamma: \bar{U} \rightarrow C(I, \mathbb{R})$ is continuous and completely continuous (which is well known to be compact restricted to bounded sets). From the choice of $U$, there is no $u \in \partial U$ such that $u=\lambda \digamma(u)$ for some $\lambda \in(0,1)$. Consequently, by Theorem 2.2, we deduce that $\nabla$ has a fixed point $u \in \bar{U}$ which is a solution of the problem (1.1). This completes the proof.

Theorem 2.4 Assume that there exist constants $0 \leq c<1 / \Delta_{2}$ and $N>0$ such that $|f(t, u)| \leq \frac{c}{T^{2}}|u|+N$ for all $t L I, u L C(I, \mathbb{R})$, where

$$
\Delta_{2}=\frac{1}{1+q}+\frac{|\eta(1+\eta q)|}{(1+q)(\eta-1)^{2}}+\left|\frac{\eta}{\eta-1}\right| .
$$

Then the BVP (1.1) has at least one solution.

Proof. We define $\digamma: \mathcal{C} \rightarrow \mathcal{C}$ as in (2.10). As in Theorem 2.3, using the assumption $|f(t, u)| \leq \frac{c}{T^{2}}|u|+N \quad$ a special form of the condition $\left(H_{1}\right)$ with $p(t)=1$, $\left.\psi(|u|)=\frac{c}{T^{2}}|u|+N\right)$, we can prove that $\otimes$ is completely continuous. 
Assume that $u=\lambda \digamma u$ for some $\lambda L[0,1]$. For all $t L I$ we have

$$
\begin{aligned}
|u(t)|= & |\lambda \digamma u(t)| \\
= & \mid \int_{0}^{t}(t-q s) f(s, u(s)) d_{q} s \\
& +\frac{\eta}{(\eta-1)^{2}} \int_{0}^{T}[T+(1-\eta)(t-q s)] f(s, u(s)) d_{q} s \\
\leq & \left(\frac{c}{T^{2}}|u|+N\right) \Delta_{2} T^{2} \\
\leq & \Delta_{2}\left(c|u|+N T^{2}\right),
\end{aligned}
$$

which implies that

$$
\|u\| \leq \frac{N \Delta_{2} T^{2}}{1-c \Delta_{2}} .
$$

Letting

$$
R=\frac{N \Delta_{2} T^{2}}{1-c \Delta_{2}}+1
$$

we have $\|u\| \leq R$. Consequently, by the nonlinear alternative of Leray-Schauder type [24], this completes the proof.

For next theorem, we need the following fixed point theorem [25].

Theorem 2.5 Let $\Omega$ be an open bounded subset of a Banach space $E$ with $\mathrm{L} \Omega$ and $B: \bar{\Omega} \rightarrow E$ be a compact operator. Then $B$ has a fixed point in $\bar{\Omega}$ provided $\|B u-u\|^{2} \geq$ $\|B u\|^{2}-\|u\|^{2}, u \in \partial \Omega$.

Theorem 2.6 Assume that there exists a positive constant $M_{1}$ such that

$$
|f(t, u)| \leq \frac{M_{1}}{\Lambda_{1}}, \quad \forall t \in I, \quad u \in\left[-M_{1}, M_{1}\right]
$$

where $\Lambda_{1}$ is given by (2.9). Then the BVP (1.1) has at least one solution.

Proof. Let us define $\Gamma=\left\{u \in C(I, \mathbb{R}):\|u\|<M_{1}\right\}$. As in Theorem 2.3, using the assumption on $f(t, u)$, it can be shown that $\nabla$ is completely continuous. Thus, in view of Theorem 2.5, we just need to show that

$$
\|\digamma u\| \leq\|u\|, \quad \forall u \in \partial \Gamma .
$$

For all $t \in I, u \in \partial \Gamma$, we have

$$
\begin{aligned}
|\digamma u(t)|= & \mid \int_{0}^{t}(t-q s) f(s, u(s)) d_{q} s \\
& +\frac{\eta}{(\eta-1)^{2}} \int_{0}^{T}[T+(1-\eta)(t-q s)] f(s, u(s)) d_{q} s \mid \\
\leq & \left|\int_{0}^{t}(t-q s) f(s, u(s)) d_{q} s\right| \\
& +\left|\frac{\eta}{(\eta-1)^{2}} \int_{0}^{T}[T+(1-\eta)(t-q s)] f(s, u(s)) d_{q} s\right| \\
\leq & \frac{M_{1}}{\Lambda_{1}}\left|\int_{0}^{t}(t-q s) d_{q} s+\frac{\eta}{(\eta-1)^{2}} \int_{0}^{T}[T+(1-\eta)(t-q s)] d_{q} s\right|,
\end{aligned}
$$

which yields $\|\digamma u\| \leq M_{1}$. Since (2.13) holds, therefore, we obtain the result. 
Remark 2.1 In view of the assumption $|f(t, u)| \leq \frac{c}{T^{2}}\|u\|+N, u \in C(I, \mathbb{R}), t \in I$ of Theorem 24, we see that it suffices to take $M_{1} \geq \frac{N \Delta_{2} T^{2}}{1-c \Delta_{2}}$.

Before presenting the last result, we state a fixed point theorem due to Krasnoselskii [26] which is needed to prove the existence of solutions of (1.1).

Theorem 2.7 (Krasnoselskii) Let $\Omega$ be a closed convex, bounded and nonempty subset of a Banach space $X$. Let $\digamma_{1}, \digamma_{2}$ be the operators such that: (i) $\digamma_{1} x+\digamma_{2} y \in \Omega$ whenever $x, y \_\Omega$; (ii) $\digamma_{1}$ is compact and continuous; (iii) $\digamma_{2}$ is a contraction mapping. Then there exists $Z \Omega$ such that $z=\digamma_{1} z+\digamma_{2} z$.

Theorem 2.8 Assume that $f: I \times \mathbb{R} \rightarrow \mathbb{R}$ is a continuous function satisfying $\left(H_{0}\right)$ and the following assumption holds:

$\left(H_{3}\right)|f(t, u)| \leq \mu(t), \forall(t, u) \in I \times \mathbb{R}$, and $\mu \in C\left(I, \mathbb{R}_{+}\right)$.

If

$$
\left(\frac{|\eta(1+\eta q)|}{(1+q)(\eta-1)^{2}}+\left|\frac{\eta}{\eta-1}\right|\right) L T^{2}<1,
$$

then the boundary value problem (1.1) has at least one solution on I.

Proof. With $\max _{t \in I}|\mu(t)|=\|\mu\|$, let us fix $r \geq\|\mu\| \Lambda_{1}\left(\Lambda_{1}\right.$ is given by (2.9)) and define $B_{r}=\{u \in \mathcal{C}:\|u\| \leq r\}$.

Define the operators $\digamma_{1} u$ and $\digamma_{2} u$ on the set $B_{r}$ as

$$
\begin{aligned}
& \digamma_{1} u(t)=\int_{0}^{t}(t-q s) f(s, u(s)) d_{q} s, \\
& \digamma_{2} u(t)=\frac{\eta}{(\eta-1)^{2}} \int_{0}^{T}[T+(1-\eta)(t-q s)] f(s, u(s)) d_{q} s .
\end{aligned}
$$

For $u, v \in B_{r}$, we have

$$
\left\|\digamma_{1} u+\digamma_{2} v\right\| \leq\|\mu\|\left(\frac{1}{1+q}+\frac{\mid \eta(1+\eta q \mid}{(1+q)(\eta-1)^{2}}+\left|\frac{\eta}{\eta-1}\right|\right) T^{2}=\|\mu\| \Lambda_{1} \leq r,
$$

which implies that $\digamma_{1} u+\digamma_{2} v \in B_{r}$. In view of the condition (2.14), it follows that $\digamma_{2}$ is a contraction mapping. Continuity of $f$ together with the assumption $\left(H_{3}\right)$ implies that the operator $\digamma_{1}$ is continuous and uniformly bounded on $B_{r}$. Let us define $\sup _{(t, u) \in I \times B r}|f(t, u)|=f_{\max }<\infty$. Then, for $t_{1}, t_{2} \in I$ with $t_{2}<t_{1}$ and $u \in B_{r}$, we have

$$
\begin{aligned}
\left\|\left(\digamma_{1} u\right)\left(t_{1}\right)-\left(\digamma_{1} u\right)\left(t_{2}\right)\right\|= & \sup _{(., u) \in I \times B r} \mid \int_{0}^{t_{1}}\left(t_{1}-q s\right) f(s, u(s)) d_{q} s \\
& -\int_{0}^{t_{2}}\left(t_{2}-q s\right) f(s, u(s)) d_{q} s \mid \\
= & \sup _{(., u) \in I \times B r} \mid \int_{0}^{t_{1}}\left(t_{1}-t_{2}\right) f(s, u(s)) d_{q} s \\
& +\int_{t_{2}}^{t_{1}}\left(t_{2}-q s\right) f(s, u(s)) d_{q} s \mid \\
\leq & f_{\max }\left(\int_{0}^{t_{1}}\left(t_{1}-t_{2}\right) d_{q} s+\int_{t_{2}}^{t_{1}}\left|t_{2}-q s\right| d_{q} s\right),
\end{aligned}
$$


which is independent of $u$ and tends to zero as $t_{2}-t_{1} \rightarrow 0$. So $\digamma_{1}$ is relatively compact on $B_{r}$. Hence, by the Arzelá-Ascoli Theorem, $\digamma_{1}$ is compact on $B_{r}$. Thus all the assumptions of Theorem 2.7 are satisfied and the conclusion of Theorem 2.7 implies that the boundary value problem (1.1) has at least one solution on $I$. This completes the proof.

\section{Examples}

Example 3.1. Consider

$$
\left\{\begin{array}{c}
D_{\frac{1}{2}}^{2} u(t)=L\left(t^{2}+\cos t+1+\tan ^{-1} u(t)\right), \quad 0 \leq t \leq 1, L>0, \\
u(0)=\frac{1}{3} u(1), \quad D_{\frac{1}{2}} u(0)=\frac{1}{3} D_{\frac{1}{2}} u(1) .
\end{array}\right.
$$

Here, $f(t, u)=L\left(t^{2}+\cos t+1+\tan ^{-1} u\right), L$ to be fixed later, $q=1 / 2, \eta=1 / 3$ and $T=$ 1. Clearly

$$
|f(t, u)-f(t, v)| \leq L\left|\tan ^{-1} u-\tan ^{-1} v\right| \leq L|u-v|
$$

and

$$
\Lambda_{1}=\left(\frac{1}{1+q}+\frac{|\eta(1+\eta q)|}{(1+q)(\eta-1)^{2}}+\left|\frac{\eta}{\eta-1}\right|\right) T^{2}=\frac{21}{12} .
$$

For $L<\frac{1}{\Lambda_{1}}=\frac{12}{21}$, it follows by Theorem 2.1 that the problem (3.1) has a unique solution.

Example 3.2. Consider the following problem

$$
\left\{\begin{array}{c}
D_{\frac{1}{2}}^{2} u(t)=\frac{c}{(2 \pi)} \sin (2 \pi u(t))+\frac{|u(t)|}{2(1+|u(t)|)}+\frac{1}{2}, 0 \leq t \leq 1, \quad c \geq 0 \\
u(0)=\frac{1}{3} u(1), \quad D_{\frac{1}{2}} u(0)=\frac{1}{3} D_{\frac{1}{2}} u(1) .
\end{array}\right.
$$

Here $q=1 / 2, \eta=1 / 3, T=1$, and $c$ will be fixed later. Observe that

$$
|f(t, u)|=\left|\frac{c}{(2 \pi)} \sin (2 \pi u)+\frac{|u(t)|}{2(1+|u(t)|)}+\frac{1}{2}\right| \leq c|u|+1,
$$

and $\Delta_{2}=\frac{21}{12}$. Obviously $N=1$ and we can choose $c<\frac{1}{\Delta_{2}}=\frac{12}{21}$. Thus, the conclusion of Theorem 2.4 applies to the problem (3.2).

Remark. By fixing $\eta$ in the results of this paper, we can obtain some special new results for $q$-difference equations. For instance, our results correspond to the ones for second-order $q$-difference equations with anti-periodic boundary conditions $(u(0)=-u$ (T), $\left.D_{q} u(0)=-D_{q} u(T)\right)$ for $\eta=-1$, and the results for an initial value problem of second-order $q$-difference equations follow for $\eta=0$. Furthermore, the classical results for second order boundary value problems with non-separated boundary conditions follow in the limit $q \rightarrow 1^{-}$. 
Acknowledgements

The authors are grateful to the reviewers for their useful comments. The research of B. Ahmad and A. Alsaedi was supported by Deanship of Scientific Research (DSR), King Abdulaziz University, Jeddah, Saudi Arabia.

\section{Author details}

'Department of Mathematics, Faculty of science, King Abdulaziz University, P.O. Box 80203, Jeddah 21589, Saudi Arabia ${ }^{2}$ Department of Mathematics, University of Ioannina, 45110 loannina, Greece

\section{Authors' contributions}

Each of the authors, BA, AA and SKN contributed to each part of this work equally and read and approved the final version of the manuscript.

\section{Competing interests}

The authors declare that they have no competing interests.

\section{Received: 1 December 2011 Accepted: 20 March 2012 Published: 20 March 2012}

\section{References}

1. Jackson, FH: On q-difference equations. American J Math. 32, 305-314 (1910). doi:10.2307/2370183

2. Carmichael, RD: The general theory of linear q-difference equations. American J Math. 34, 147-168 (1912). doi:10.2307/ 2369887

3. Mason, TE: On properties of the solutions of linear q-difference equations with entire function coefficients. American Math. 37, 439-444 (1915). doi:10.2307/2370216

4. Adams, CR: On the linear ordinary q-difference equation. Annals Math. 30, 195-205 (1928). doi:10.2307/1968274

5. Finkelstein, R, Marcus, E: Transformation theory of the q-oscillator. J Math Phys. 36, 2652-2672 (1995). doi:10.1063/ 1.531057

6. Finkelstein, R: The q-Coulomb problem. J Math Phys. 37, 2628-2636 (1996). doi:10.1063/1.531532

7. Floreanini, R, Vinet, L: Automorphisms of the q-oscillator algebra and basic orthogonal polynomials. Phys Lett A. 180 , 393-401 (1993). doi:10.1016/0375-9601(93)90289-C

8. Floreanini, R, Vinet, L: Symmetries of the q-difference heat equation. Lett Math Phys. 32, 37-44 (1994). doi:10.1007/ BF00761122

9. Floreanini, R, Vinet, L: Quantum symmetries of q-difference equations. J Math Phys. 36, 3134-3156 (1995). doi:10.1063/ 1.531017

10. Freund, PGO, Zabrodin, AV: The spectral problem for the q-Knizhnik-Zamolodchikov equation and continuous q-Jacobi polynomials. Comm Math Phys. 173, 17-42 (1995). doi:10.1007/BF02100180

11. Han, GN, Zeng, J: On a q-sequence that generalizes the median Genocchi numbers. Ann Sci Math Quebec. 23, 63-72 (1999)

12. Jaulent, M, Miodek, I: Nonlinear evolution equations associated with 'energy-dependent Schrodinger potentials'. Lett Math Phys. 1, 243-250 (1976). doi:10.1007/BF00417611

13. Ernst, T: A new notation for q-calculus and a new q-Taylor formula. Department of Mathematics, Uppsala University (1999). U.U.D.M. Report 1999:25, ISSN 1101-3591

14. Gasper, G, Rahman, M: Basic Hypergeometric Series. Cambridge University Press, Cambridge (1990)

15. Kac, V, Cheung, P: Quantum Calculus. Springer, New York (2002)

16. Bangerezako, G: Variational q-calculus. J Math Anal Appl. 289, 650-665 (2004). doi:10.1016/j.jmaa.2003.09.004

17. Dobrogowska, A, Odzijewicz, A: Second order q-difference equations solvable by factorization method. J Comput Appl Math. 193, 319-346 (2006). doi:10.1016/j.cam.2005.06.009

18. Gasper, G, Rahman, M: Some systems of multivariable orthogonal q-Racah polynomials. Ramanujan J. 13, 389-405 (2007). doi:10.1007/s11139-006-0259-8

19. Annaby, MH, Mansour, ZS: q-Taylor and interpolation series for Jackson q-difference operators. J Math Anal Appl. 344, 472-483 (2008). doi:10.1016/j.jmaa.2008.02.033

20. Ismail, MEH, Simeonov, P: q-difference operators for orthogonal polynomials. J Computat Appl Math. 233, 749-761 (2009). doi:10.1016/j.cam.2009.02.044

21. El-Shahed, M, Hassan, HA: Positive solutions of q-difference equation. Proc Amer Math Soc. 138, 1733-1738 (2010)

22. Ahmad, B, Ntouyas, SK: Boundary value problems for q-difference inclusions. Abstr Appl Anal2011, 15. Article ID 292860

23. Ahmad, B: Boundary-value problems for nonlinear third-order q-difference equations. Electron J Diff Equ. 2011(94):1-7 (2011)

24. Granas, A, Dugundii, J: Fixed Point Theory. Springer-Verlag, New York (2005)

25. Altman, M: A fixed point theorem in Banach space. Bull Polish Acad Sci. 5, 19-22 (1957)

26. Smart, DR: Fixed Point Theorems. Cambridge University Press (1980)

doi:10.1186/1687-1847-2012-35

Cite this article as: Ahmad et al:: A study of second-order q-difference equations with boundary conditions.

Advances in Difference Equations 2012 2012:35. 